\title{
Efficacy of adjunctive treatment with trimetazidine for fragmented QRS among patients with ischemic heart failure: a propensity score match analysis
}

\author{
Selçuk Kanat๑, Barış Şensoy॰ \\ Department of Cardiology, University of Health Sciences, Bursa Yüksek Ihtisas Training and Research Hospital, Bursa, Turkey
}

DOI: $10.18621 /$ eurj.397369

\begin{abstract}
Objectives: Fragmented QRS (fQRS) is an indicator of nonhomogeneous ventricular activity caused by myocardial ischemia and fibrosis. The anti-ischemic agent trimetazidine (TMZ) added to pharmacological treatment appears to have positive effects on cardiac parameters of patients with ischemic heart failure. We aimed to investigate the relationship of fQRS with adjunctive TMZ therapy in ischemic HF patients.

Methods: Four hundred eighty-nine consecutive ambulatory ischemic patients with heart failure eligible for our study were recruited for the study. A 12-lead electrocardiogram with standard chest and limb leads was used to evaluate the presence of fQRS. Further patients were divided into groups according to adjunctive TMZ treatment and fQRS presence. Confounding factors were adjusted by propensity score matching and multivariate logistic regression analysis.

Results: One hundred ninety-seven (40.3\%) patients had fQRS on their ECGs and 235 (48.1\%) patients were on adjunctive treatment with trimetazidine. Compared to patients without fQRS, patients with fQRS had lower left ventricular ejection fraction (LVEF), higher NYHA classes and more frequent mineralocorticoid receptor antagonist and diuretic usage $(p<0.05)$. Add-on treatment with TMZ was independently associated with fQRS presence (OR, 0.45; (95\% CI, 0.29-0.70); $p<0.0001)$.

Conclusion: According to conventional therapy, adjunctive treatment with TMZ among ischemic heart failure patients can be associated with fQRS in 12-lead ECG independent of LVEF.
\end{abstract}

Keywords: Adjunctive treatment, fragmented QRS, ischemic heart failure, myocardial fibrosis, trimetazidine

Received: February 21, 2018; Accepted: August 1, 2018; Published Online: January 10, 2019

A lthough advances in acute cardiac care have improved survival after myocardial infarction (MI), there has been a rise in frequency of patients who have a poor prognosis due to the ineluctable progression of contractile dysfunction and left ventricular (LV) enlargement despite the recent introduction of many therapies [1]. As scientific evidence has shown that in addition to hemodynamic alterations, deep changes occur in the metabolism of failing heart; metabolic modulation has become an attractive therapeutic approach to heart failure (HF) [2-5]. Shifting metabolism away from a preference for free fatty acid toward more carbohydrate oxidation by Trimetazidine (1-[2,3,4trimethoxybenzyl] piperazine dihydrochloride, TMZ) has suggested to be the mechanism for the beneficial effects of TMZ given to ischemic HF patients as an add-on therapy. TMZ was observed to have a beneficial effect on LV remodeling, cardiac function, exer-

Address for correspondence: Selçuk Kanat, MD., Assistant Professor, University of Health Sciences, Bursa Yüksek Ihtisas Training and Research Hospital, Department of Cardiology, Bursa, Turkey 
cise tolerance, endothelium-dependent relaxation and electrophysiological properties for risk of major arrhythmias when added to conventional pharmacological treatment in patients with ischemic HF [2, 6-8]. Recently add-on therapy with TMZ have demonstrated to reduce mortality in these patients [1].

Several clinical parameters have shown association to cardiac death and HF progression including older age, prior MI, and diabetes mellitus [9]. Fragmentation in the QRS complex (fQRS) in 12-lead ECG has also been shown for prediction of cardiac events in patients with coronary artery disease (CAD) [10]. The fQRS is a result of myocardial scarring or ischemia that causes heterogeneous ventricular activation and dyssynchronous contraction $[11,12]$. The effect of adjunctive treatment with trimetazidine on the presence of $\mathrm{fQRS}$ in ischemic HF patients is obscure. So, in this study, we aimed to investigate the association of add-on therapy with TMZ with the presence and extent of $\mathrm{fQRS}$ in patients with ischemic HF.

\section{METHODS}

The study population included patients with heart failure who were admitted to the cardiology outpatient clinics for ischemic heart disease and were under treatment with optimal, regular medication modalities for at least 6 months. The etiology of the heart failure was coronary artery disease documented by coronary angiography and a comprehensive echocardiographic examination, including M-mode, two-dimensional and Doppler echocardiography in all of the included patients [13]. Information was obtained by document review and patient interview following patient consent. Patients' medical notes provided patient details, past medical history, laboratory investigations, drug treatment on admission, and other relevant information such as adverse effects and compliance. Patients who have irregular trimetazidine usage (previous usage with cessation or not taken properly), mitral, aort, tricuspid and pulmoner stenosis or other severe valvular heart disease, non-ischemic dilated cardiomyopathy, left ventricular ejection fraction (LVEF) $>50 \%$ in echocardiography and New York Heart Association class (NYHA) IV were excluded from the study. Other exclusion criteria were; patients with acute myocardial infarction in the previous 3 months, with atrial fibrillation, chronic lung disease or any systemic disorder, complete or incomplete bundle branch block and pacemaker rhythm on ECG and use of additional antiarrhythmic drugs other than beta blockers.

All subjects were screened with a questionnaire. Demographic information, comorbidities, medications, symptoms, and history of trimetazidine therapy of the patients were obtained. Hypertension (HT) (systolic or diastolic blood pressure $>140$ and $90 \mathrm{~mm} \mathrm{Hg}$, respectively, or pharmacological therapy with antihypertensive drugs), diabetes mellitus (DM) (fasting glucose plasma concentrations $>126 \mathrm{mg} / \mathrm{dL}$ or pharmacological therapy with antidiabetic drugs or insulin), hyperlipidemia ( low-density lipoprotein (LDL) cholesterol levels $\geq 130 \mathrm{mg} / \mathrm{dl}$ or being treated with lipid-lowering medication) were considered definitions. Smoking was defined as at least 20 cigarettes per month for more than 6 months.

A 12-lead electrocardiogram with standard chest and limb leads was used to evaluate the presence of fQRS. The paper speed and amplitude were set to 25 $\mathrm{mm} / \mathrm{s}$ and $10 \mathrm{~mm} / \mathrm{mV}$, respectively. The lowfrequency cutoff (high-pass filter) was set at $0.16 \mathrm{~Hz}$ and high-frequency cut off (low-pass filters) was set at $100 \mathrm{~Hz}$. All ECGs were interpreted by two cardiologists who had no knowledge of the patients' clinical information. Fragmented QRS was defined as the QRS complexes with the presence of an additional $R$ wave ( $R$ ') or notching in the nadir of the $R$ wave or the $\mathrm{S}$ wave, or the presence of $>1 \mathrm{R}^{\prime}$ in two contiguous leads, corresponding to a major coronary territory. The study was approved by hospital Ethics Committee and patients gave their written informed consent.

\section{Statistical Analysis}

The SPSS statistical software (SPSS 17.0 for Windows, Inc., Chicago, IL, USA) was used for data analysis. Quantitative data were presented as mean \pm standard deviation or median and interquartile range, and categorical variables presented as percentages. After testing data for normal distribution by using Shapiro-Wilk test, Student's t-test or Mann-Whitney $\mathrm{U}$ test were used to compare continuous variables. Chi-square test or Fisher's exact test, as appropriate, were used to identify statistically significant differences for categorical variables. To evaluate the 
correlations between fQRS and clinical presentation, we used the Spearman's $\rho$ correlation analysis. To minimize the confounding effect of possible factors and to obtain the best balance among groups, we performed a multivariate logistic regression model based on the significant demographic, clinical and treatment characteristic variables [14]. Logistic regression analysis was used to examine the associations between fragmented QRS, additional treatment with trimetazidine and other variables. Variables with a $p$ value of $<0.1$ in univariate logistic regression analysis were included in a multivariate logistic regression model. Statistical significance was defined as $p<0.05$. Statistical tests were two-sided.

Table1. Baseline characteristics of patients with and without fragmented QRS

\begin{tabular}{|c|c|c|c|c|}
\hline & $\begin{array}{c}\text { All } \\
\mathrm{n}=\mathbf{4 8 9}\end{array}$ & $\begin{array}{c}\text { fQRS (+) } \\
\mathbf{n}=197\end{array}$ & $\begin{array}{c}\text { fQRS (-) } \\
\text { n = 292 }\end{array}$ & $p$ value \\
\hline Age, years & $61.1 \pm 8.4$ & $62.5 \pm 8.8$ & $60.1 \pm 8.1$ & 0.003 \\
\hline Age $>65$ years, $n(\%)$ & $133(27)$ & $80(41)$ & $53(18)$ & $<0.0001$ \\
\hline Male, n (\%) & $327(67)$ & $154(78)$ & $173(59)$ & $<0.0001$ \\
\hline BMI, $\mathrm{kg} / \mathrm{m}^{2}$ & $27.2 \pm 3.2$ & $26.9 \pm 2.9$ & $27.4 \pm 3.5$ & 0.15 \\
\hline LVEF, \% & $41.4 \pm 8.3$ & $39.3 \pm 7.7$ & $42.8 \pm 6.3$ & $<0.0001$ \\
\hline LVH, n (\%) & $208(43)$ & $88(45)$ & $120(41)$ & 0.43 \\
\hline NYHA, n (\%) & & & & $<0.0001$ \\
\hline I & $337(69)$ & $88(45)$ & $249(85)$ & \\
\hline II & $126(26)$ & $83(42)$ & $43(15)$ & \\
\hline III & $26(5)$ & $26(13)$ & $0(0)$ & \\
\hline DM, n (\%) & $143(29)$ & $73(37)$ & $70(24)$ & 0.002 \\
\hline HT, n (\%) & $285(58)$ & $141(72)$ & $144(49)$ & $<0.0001$ \\
\hline Dyslipidemia, n (\%) & $461(94)$ & $183(93)$ & $278(95)$ & 0.28 \\
\hline Smoking, n (\%) & $349(71)$ & $136(69)$ & $213(73)$ & 0.35 \\
\hline ACE-I, n (\%) & $299(61)$ & $125(64)$ & $174(60)$ & 0.39 \\
\hline ARB, n (\%) & $103(21)$ & $39(20)$ & $64(22)$ & 0.57 \\
\hline Diuretic, n (\%) & $199(41)$ & $117(59)$ & $82(28)$ & $<0.0001$ \\
\hline MRA, n (\%) & $124(25)$ & $79(40)$ & $45(15)$ & $<0.0001$ \\
\hline$\beta$-Blocker, n (\%) & $324(66)$ & $121(61)$ & $203(69)$ & 0.07 \\
\hline Digoxin, n (\%) & $63(13)$ & $20(10)$ & $43(15)$ & 0.14 \\
\hline ASA, n (\%) & $446(91)$ & $177(90)$ & $269(92)$ & 0.38 \\
\hline Statin, n (\%) & $309(63)$ & $133(68)$ & $176(60)$ & 0.11 \\
\hline Insulin/OAD, n (\%) & $130(27)$ & $68(34)$ & $62(21)$ & 0.001 \\
\hline $\mathrm{HbA1c}, \%$ & $5.2(5.1-6.2)$ & $5.7(5.2-6.3)$ & $5.1(4.8-5.5)$ & $<0.0001$ \\
\hline eGFR, $\mathrm{mL} / \mathrm{min}$ per $1.73 \mathrm{~m}^{2}$ & $85.1 \pm 28.9$ & $83.5 \pm 23.1$ & $86.1 \pm 32.3$ & 0.36 \\
\hline No. leads with fQRS & $0(0-3)$ & $4(3-4)$ & $0(0-0)$ & $<0.0001$ \\
\hline TMZ Treatment, n (\%) & $235(48)$ & $72(36)$ & $163(56)$ & $<0.0001$ \\
\hline
\end{tabular}

ACE-I $=$ angiotensin-converting enzyme inhibitor, ARB $=$ angiotensin receptor blocker, ASA $=$ acetylsalicylic acid, $\mathrm{BMI}=$ body mass index, $\mathrm{DM}=$ diabetes mellitus, $\mathrm{eGFR}=$ estimated glomerular filtration rate, $\mathrm{fQRS}=$ fragmented QRS complex, HbA1c = hemoglobin A1c, HT = hypertension, $\mathrm{LVEF}=$ left ventricular ejection fraction $=\mathrm{LVH}=$ left ventricular hypertrophy, MRA $=$ mineralocorticoid receptor antagonist, NYHA $=$ New York Heart Association class, $\mathrm{OAD}=$ oral antidiabetic, $\mathrm{TMZ}=$ Trimetazidine 


\section{RESULTS}

Of 489 consecutive patients recruited for the study, 327 (67\%) were male and 133 (27\%) were more than 65 years old. Mean age of the patients was 61.1 \pm 8.4. Among the participants, 285 (58\%) had hypertension, 143 (29\%) had diabetes mellitus, and
461 (94\%) had hyperlipidemia. One hundred and ninety seven $(40.3 \%)$ patients had fQRS on their ECGs. Compared to patients without fQRS, patients with fQRS had lower LVEF, higher NYHA classes and more frequent mineralocorticoid receptor antagonist (MRA) and diuretic usage. Also adjunctive treatment with Trimetazidine was significantly lower in patients

Table 2. Baseline characteristics of the study population according to the treatment modality.

\begin{tabular}{|c|c|c|c|c|}
\hline & $\begin{array}{c}\text { All } \\
n=489\end{array}$ & $\begin{array}{l}\text { Add-on treatment with } \\
\text { TMZ } \\
\mathbf{n}=\mathbf{2 3 5}\end{array}$ & $\begin{array}{c}\text { Conventional } \\
\text { treatment } \\
n=254\end{array}$ & $p$ value \\
\hline Age, years & $61.1 \pm 8.4$ & $61.5 \pm 8.5$ & $60.7 \pm 8.4$ & 0.29 \\
\hline Age $>65$ years, $n(\%)$ & $133(27)$ & $69(29)$ & $64(25)$ & 0.30 \\
\hline Male, n (\%) & $346(71)$ & $164(70)$ & $182(72)$ & 0.65 \\
\hline BMI, kg/m² & $27.2 \pm 3.2$ & $27.5 \pm 3.3$ & $26.9 \pm 3.2$ & 0.07 \\
\hline LVEF, \% & $41.4 \pm 8.3$ & $44.8 \pm 4.9$ & $38.2 \pm 7.6$ & $<0.0001$ \\
\hline LVH, n (\%) & $208(43)$ & $98(42)$ & $110(43)$ & 0.72 \\
\hline NYHA, n (\%) & & & & $<0.0001$ \\
\hline I & 337 (69) & $194(83)$ & $143(56)$ & \\
\hline II & $126(26)$ & $41(17)$ & $85(34)$ & \\
\hline III & $26(5)$ & $0(0)$ & $26(10)$ & \\
\hline $\mathrm{DM}, \mathrm{n}(\%)$ & $143(29)$ & $69(29)$ & $74(29)$ & 0.96 \\
\hline $\mathrm{HT}, \mathrm{n}(\%)$ & $285(58)$ & $138(59)$ & $147(58)$ & 0.85 \\
\hline Dyslipidemia, n (\%) & $461(94)$ & $220(94)$ & $241(95)$ & 0.55 \\
\hline Smoking, n (\%) & $349(71)$ & $176(75)$ & $173(68)$ & 0.10 \\
\hline ACE-I, n (\%) & $299(61)$ & $143(61)$ & $156(61)$ & 0.90 \\
\hline $\mathrm{ARB}, \mathrm{n}(\%)$ & $103(21)$ & $63(27)$ & $40(16)$ & 0.003 \\
\hline Diuretic, n (\%) & $199(41)$ & $73(31)$ & $126(50)$ & $<0.0001$ \\
\hline MRA, n (\%) & $124(25)$ & $33(14)$ & $91(36)$ & $<0.0001$ \\
\hline$\beta$-Blocker, n (\%) & $324(66)$ & $171(73)$ & $153(60)$ & 0.003 \\
\hline Digoxin, n (\%) & $63(13)$ & $38(16)$ & $25(10)$ & 0.037 \\
\hline ASA, n (\%) & $446(91)$ & $212(90)$ & $234(92)$ & 0.46 \\
\hline Statin, n (\%) & $309(63)$ & $139(59)$ & $170(67)$ & 0.08 \\
\hline Insulin/OAD, n (\%) & $130(27)$ & $61(26)$ & $69(27)$ & 0.76 \\
\hline $\mathrm{HbA1c}, \%$ & $5.2(5.1-6.2)$ & $5.2(5.0-6.2)$ & $5.3(5.1-6.3)$ & 0.27 \\
\hline eGFR, $\mathrm{mL} / \mathrm{min}$ per $1.73 \mathrm{~m}^{2}$ & $85.1 \pm 28.9$ & $83.9 \pm 28.6$ & $85.9 \pm 29.2$ & 0.45 \\
\hline fQRS, n $(\%)$ & $197(40)$ & $72(31)$ & $125(49)$ & $<0.0001$ \\
\hline No. leads with fQRS & $0(0-3)$ & $0(0-2)$ & $0(0-4)$ & $<0.0001$ \\
\hline
\end{tabular}

ACE-I = angiotensin-converting enzyme inhibitor, ARB $=$ angiotensin receptor blocker, ASA = acetylsalicylic acid, $\mathrm{BMI}=$ body mass index, $\mathrm{DM}=$ diabetes mellitus, eGFR = estimated glomerular filtration rate, $\mathrm{fQRS}=$ fragmented QRS complex, HbA1c = hemoglobin A1c, HT = hypertension, LVEF = left ventricular ejection fraction, $\mathrm{LVH}=$ left ventricular hypertrophy, $\mathrm{MRA}=$ mineralocorticoid receptor antagonist, NYHA $=$ New York Heart Association class, $\mathrm{OAD}=$ oral antidiabetic, $\mathrm{TMZ}=$ Trimetazidine 
Table 3. Characteristics of our matched study group according to the treatment modality

\begin{tabular}{lcccc}
\hline & $\begin{array}{c}\text { All } \\
\mathbf{n}=\mathbf{1 7 6}\end{array}$ & $\begin{array}{c}\text { Add-on treatment with } \\
\mathbf{T M Z} \\
\mathbf{n = 8 8}\end{array}$ & $\begin{array}{c}\text { Conventional } \\
\text { treatment } \\
\mathbf{n = 8 8}\end{array}$ & $\boldsymbol{p}$ value \\
\hline Age $>$ 65 years, n (\%) & $40(23)$ & $20(23)$ & $20(23)$ & 1 \\
LVEF, \% & $41.3 \pm 7.8$ & $43.5 \pm 8.0$ & $39.2 \pm 7.1$ & $<\mathbf{0 . 0 0 0 1}$ \\
NYHA, n (\%) & & & & $\mathbf{0 . 0 0 3}$ \\
I & $129(73)$ & $74(84)$ & $55(63)$ & \\
II & $44(25)$ & $14(16)$ & $30(34)$ & \\
III & $3(2)$ & $0(0)$ & $3(3)$ & \\
LVEF $<30 \%$, n $(\%)$ & $28(16)$ & $11(13)$ & $17(19)$ & 0.22 \\
LVH, n $(\%)$ & $80(46)$ & $43(49)$ & $37(42)$ & 0.36 \\
fQRS, n $(\%)$ & $62(35)$ & $24(27)$ & $38(43)$ & $\mathbf{0 . 0 3}$ \\
No. leads with fQRS & $0(0-3)$ & $0(0-2)$ & $0(0-3.5)$ & $\mathbf{0 . 0 5}$ \\
\hline
\end{tabular}

fQRS = fragmented QRS complex, LVEF = left ventricular ejection fraction, LVH = left ventricular hypertrophy, NYHA $=$ New York Heart Association class, TMZ $=$ Trimetazidine

with fQRS according to non-fQRS group. While HT and DM were more frequent in patients with fQRS, frequency of left ventricular hypertrophy (LVH) did not differ significantly between groups (Table 1). In subgroup of patients with $\mathrm{EF} \leq 30 \%$ prevalence of
fQRS and number of leads with fQRS didn't differed between the treatment modality groups ( 8 patients $(40 \%)$ vs. 30 patients (48\%) and $0(0-3)$ vs. $0(0-4) ; p$ $>0.05$ respectively).

Number of leads with fQRS on ECG was

Table 4. Association of fragmented QRS with multiple variables in univariate and multivariate logistic regression analyses.

\begin{tabular}{lcccc}
\hline Variables & $\begin{array}{c}\text { Univariate } \\
\text { OR (95\% CI) }\end{array}$ & $\boldsymbol{p}$ value & $\begin{array}{c}\text { Multivariate } \\
\text { OR (95\% CI)* }\end{array}$ & $p$ value \\
\hline Age $>$ 65 years & $3.08(2.04-4.65)$ & $<\mathbf{0 . 0 0 0 1}$ & $2.47(1.53-3.99)$ & $<\mathbf{0 . 0 0 0 1}$ \\
Male Gender & $2.48(1.67-3.70)$ & $<\mathbf{0 . 0 0 0 1}$ & $2.43(1.56-3.79)$ & $<\mathbf{0 . 0 0 0 1}$ \\
LVEF & $1.05(1.03-1.08)$ & $<\mathbf{0 . 0 0 0 1}$ & $1.02(0.99-1.05)$ & 0.18 \\
LVEF $<30 \%$ & $1.3(0.8-2.1)$ & 0.26 & - & - \\
LVH & $1.2(0.8-1.7)$ & 0.43 & - & - \\
DM & $1.87(1.26-2.77)$ & $\mathbf{0 . 0 0 2}$ & $1.33(0.83-2.14)$ & 0.24 \\
HT & $2.59(1.76-3.80)$ & $<\mathbf{0 . 0 0 0 1}$ & $1.77(1.11-2.83)$ & $\mathbf{0 . 0 2}$ \\
ACE-I & $1.18(0.81-1.71)$ & 0.39 & - & - \\
ARB & $0.88(0.56-1.37)$ & 0.58 & - & - \\
$\beta$-Blocker & $0.70(0.48-1.02)$ & 0.07 & $0.66(0.43-1.00)$ & 0.06 \\
TMZ Treatment & $0.46(0.31-0.66)$ & $<\mathbf{0 . 0 0 0 1}$ & $0.45(0.29-0.70)$ & $<\mathbf{0 . 0 0 0 1}$ \\
\hline ACE- $=$. & & & -
\end{tabular}

ACE-I $=$ angiotensin-converting enzyme inhibitor, $\mathrm{ARB}=$ angiotensin receptor blocker, $\mathrm{CI}=$ confidence interval, $\mathrm{DM}=$ diabetes mellitus, $\mathrm{HT}=$ hypertension, $\mathrm{LVEF}=$ left ventricular ejection fraction, $\mathrm{LVH}=$ left ventricular hypertrophy, $\mathrm{OR}=$ odds ratio, $\mathrm{TMZ}=$ Trimetazidine.

*Adjusted for: Age $>65$ years, male gender, left ventricular ejection fraction, diabetes mellitus, hypertension, $\beta$ Blocker and Trimetazidine usage. 
significantly correlated with NYHA functional class $\left(r_{s}=0.47, p<0.0001\right)$, LVEF $\left(\mathrm{r} r_{s}=-0.27, p<0.0001\right)$, usage of diuretics $\left(r_{s}=0.30, p<0.0001\right)$ and MRAs $\left(r_{s}=0.33, p<0.0001\right)$.

Patients were on standard medical therapy for heart failure (HF) according to current guidelines [15]. Two hundred and thirty five (48.1\%) patients were on TMZ treatment added to conventional therapy. When patients were classified according to adjunctive TMZ treatment there were no differences between the 2 groups with regard to the specific drugs used with the exception of diuretics, MRA, $\beta$-Blocker, digoxin and angiotensin receptor blockers (ARB). Patients in the conventional treatment group had higher rates of fQRS and higher number of leads with fQRS $(p<$ 0.0001) (Table 2). After matching groups according to demographic, clinical and treatment characteristics, presence of fQRS remained significantly less frequent in adjunctive TMZ treatment group (Table 3 ). There were no differences between the groups regarding hematologic and biochemical parameters.

In stepwise multivariate logistic regression analyze, age $>65$ years, male gender, hypertension and add-on treatment with TMZ were independently associated with fQRS presence (Table 4).

\section{DISCUSSION}

Our study results showed the independent association of fQRS in 12-lead ECG with adjunctive TMZ treatment according to conventional therapy among patients with ischemic HF.

In this study, the presence of fQRS was evaluated in ambulatory ischemic systolic HF patients to investigate its relation with clinical symptoms and add-on TMZ therapy. Many studies have demonstrated that the rates of the fQRS widely range between percentages $(28 \%-54 \%)[10,11,16]$. This wide range can be explained by the differences in the patients' groups, different treatment methods and variability in the ranges of filters adopted in the ECG imaging of these studies. In this study, the frequency of fQRS was $40.1 \%$ in ischemic systolic HF patients. The extent of fQRS on ECG was correlated with NYHA functional class, and both the presence and extent of fQRS were associated with a poor overall clinical presentation besides NYHA class, including increased usage of diuretics and MRAs, and lower LVEF. Because of well documented negative correlation of LVEF with the number of leads with fQRS, we proposed that those patients with increasing number of leads with fQRS had greater myocardial scar tissue or ischemia in our study. Our suggestion was compatible with the scholars as successful myocardial reperfusion by invasive treatment had decreased the number of fQRS leads and the probability of ischemia detection by myocardial perfusion scintigrams was higher in patients whose ECGs have fQRS [17, 18]. Also, while, fQRS was found to be associated with poor functional NYHA class in hospitalized decompensated systolic heart failure patients [19]; different from our study, Cheema et al. [20] could not demonstrate a relationship between fQRS and EF in patients receiving ICDs for primary prevention due to ischemic and non-ischemic heart failure. The diffusiveness of probable residual ischemia and the differences in clinical characteristics of the enrolled patients may be likely explanations for this discrepancy.

The $\mathrm{fQRS}$ is related to a higher adverse cardiac event and decreased life span in patients with known atherosclerotic heart disease [10]. Myocardial scar and/or ischemia have been implicated in the formation of fragmentation of the QRS complex, leading to inhomogeneous ventricular activations. Different fQRS morphologies are caused by shifting QRS vector during depolarization in and around scars or myocardial ischemic areas, depending on their extent and ventricular locations $[16,21,22]$. The beneficial effects of TMZ on left ventricular systolic function and functional capacity were emphasized more markedly in patients with more severe reversible perfusion defects [23]. Although residual ischemia wasn't evaluated in our study; benefits of adjunctive metabolic treatment was indirectly supported by demonstrating the higher prevalence of the fQRS presence in ischemic HF patients with conventional treatment barring TMZ. The diagnostic accuracy of fQRS has been investigated in different studies in which they have showed that in addition to significant relation with coronary reversible ischemia $[17,18]$, fQRS was moderately sensitive but highly specific for myocardial scar [24]. The fQRS have also been defined as a predictor of arrhythmic events and 
appropriate ICD shocks in patients with cardiomyopathy who received an implantable cardioverter-defibrillator (ICD) for primary or secondary prevention of sudden cardiac death (SCD) $[16,25]$. Similarly, increased fragmentation might also serve as a marker of abnormal spread of ventricular activation leading to dyssynchronous contraction and impending HF [26]. In patients receiving cardiac resynchronization therapy for $\mathrm{HF}$, increased number of leads with fQRS predicted response to treatment [26]. Both arrhythmias and worsening HF were demonstrated to be associated with a poor prognosis and prognostic value of add-on therapy with TMZ in ischemic HF patients was determined [1, 11, 12]. Our findings reinforce this evidence by the relatively low prevalence of $\mathrm{fQRS}$ presence and higher mean EF values in add-on TMZ treatment group relative to conventional treatment group.

Of interest, patients who had higher number of leads with $\mathrm{fQRS}$ were less frequent in adjunctive TMZ treatment group relatively to the conventional treatment group. In another study carried out in ischemic HF patients, the number of leads with fQRS was an independent predictor of cardiovascular mortality or re-hospitalization for HF [27]. We failed to demonstrate a similar association between number of leads with fQRS and treatment modality in subgroup of patients with $\mathrm{EF} \leq 30 \%$, although the small number of patients in these subgroups degreases the power of statistical assessment. So, we have postulated that fQRS precisely represents the myocardial scar other than ischemia in patients with $\mathrm{EF} \leq 30 \%$ and may discriminate patients with poor prognosis.

\section{Limitations}

The limitations of the study include crosssectional and single-center design. It was non-randomized, and thus, subject to selection bias. However, we were careful to include consecutive patients and had strengthened our results by matching groups' with propensity score analysis. The fQRS was not defined in the presence of complete or incomplete bundle branch blocks or paced rhythms, and therefore in this present study these patients were excluded from the study. Also, we did not use other quantitative modalities (myocardial perfusion scanning, stress echocardiography, or magnetic resonance imaging) to show the myocardial ischemia or scarring. Evaluation of residual ischemia wasn't performed with appropriate techniques; therefore contribution of residual ischemia to the trimetazidine treatment efficiency on fQRS prevalence is obscure.

\section{CONCLUSION}

In light of the present study by finding a significant association between the clinical presentation, adjunctive treatment with TMZ and higher fQRS frequency; we suggest that the prognostic benefit of add-on TMZ treatment can be at least partly by degreasing the frequency of the fQRS on ECG. Since the presence of $\mathrm{fQRS}$ in 12-lead ECG is related to myocardial ischemia or scarring; monitoring these patients closely by myocardial perfusion scanning or magnetic resonance imaging can allow us for discriminating patients who would benefit most from invasive or non-invasive treatment strategies. Nevertheless, future prospectively designed large scale studies going deeper in this discussion are required for investigating the importance of the adjunctive Trimetazidine therapy on FQRS.

\section{Conflict of interest}

The authors disclosed no conflict of interest during the preparation or publication of this manuscript.

\section{Financing}

The authors disclosed that they did not receive any grant during conduction or writing of this study.

\section{REFERENCES}

[1] Grajek S, Michalak M. The effect of trimetazidine added to pharmacological treatment on all-cause mortality in patients with systolic heart failure. Cardiology 2015;131:22-9.

[2] Sensoy B, Canpolat U, Cay S, Ozeke O, Acikgoz K, Kus O, et al. Treatment of mechanical valve thrombosis in the right heart: overview of the requirements for the new thrombolytic protocols. Blood Coagul Fibrinolysis 2015;26:220-2.

[3] Tang WH. The metabolic approach in patients with heart failure: effects on left ventricle remodeling. Curr Pharm Des 2009; 15:850-6.

[4] Fragasso G, Salerno A, Spoladore R, Bassanelli G, Arioli F, Margonato A. Metabolic therapy of heart failure. Curr Pharm Des 2008;14:2582-91. 
[5] Lionetti V, Stanley WC, Recchia FA. Modulating fatty acid oxidation in heart failure. Cardiovasc Res 2011;90:202-9.

[6] Belardinelli R, Solenghi M, Volpe L, Purcaro A. Trimetazidine improves endothelial dysfunction in chronic heart failure: an antioxidant effect. Eur Heart J 2007;28:1102-8.

[7] El-Kady T, El-Sabban K, Gabaly M, Sabry A, Abdel-Hady S. Effects of trimetazidine on myocardial perfusion and the contractile response of chronically dysfunctional myocardium in ischemic cardiomyopathy: a 24-month study. Am J Cardiovasc Drugs 2005;5:271-8.

[8] Cera M, Salerno A, Fragasso G, Montanaro C, Gardini C, Marinosci G, et al. Beneficial electrophysiological effects of trimetazidine in patients with postischemic chronic heart failure. J Cardiovasc Pharmacol Ther 2010;15:24-30.

[9] Korhonen P, Husa T, Konttila T, Tierala I, Makijarvi M, Vaananen H, et al. Fragmented QRS in prediction of cardiac deaths and heart failure hospitalizations after myocardial infarction. Ann Noninvasive Electrocardiol 2010;15:130-7.

[10] Das MK, Saha C, El Masry H, Peng J, Dandamudi G, Mahenthiran J, et al. Fragmented QRS on a 12-lead ECG: a predictor of mortality and cardiac events in patients with coronary artery disease. Heart Rhythm 2007;4:1385-92.

[11] Das MK, Khan B, Jacob S, Kumar A, Mahenthiran J. Significance of a fragmented QRS complex versus a Q wave in patients with coronary artery disease. Circulation. 2006;113:2495-501.

[12] Mahenthiran J, Khan BR, Sawada SG, Das MK. Fragmented QRS complexes not typical of a bundle branch block: a marker of greater myocardial perfusion tomography abnormalities in coronary artery disease. J Nucl Cardiol 2007;14:347-53.

[13] Lang RM, Bierig M, Devereux RB, Flachskampf FA, Foster E, Pellikka PA, et al. Recommendations for chamber quantification: a report from the American Society of Echocardiography's Guidelines and Standards Committee and the Chamber Quantification Writing Group, developed in conjunction with the European Association of Echocardiography, a branch of the European Society of Cardiology. J Am Soc Echocardiogr 2005;18:1440-63.

[14] Rubin DB. The design versus the analysis of observational studies for causal effects: parallels with the design of randomized trials. Stat Med 2007;26:20-36.

[15] McMurray JJ, Adamopoulos S, Anker SD, Auricchio A, Bohm M, Dickstein K, et al. ESC Guidelines for the diagnosis and treatment of acute and chronic heart failure 2012: The Task Force for the Diagnosis and Treatment of Acute and Chronic Heart Failure 2012 of the European Society of Cardiology. Developed in collaboration with the Heart Failure Association (HFA) of the ESC. Eur Heart J 2012;33:1787-847.

[16] Das MK, El Masry H. Fragmented QRS and other depolarization abnormalities as a predictor of mortality and sudden cardiac death. Curr Opin Cardiol 2010;25:59-64.

[17] Ozdemir S, Tan YZ, Colkesen Y, Temiz A, Turker F, Akgoz

S. Comparison of fragmented QRS and myocardial perfusiongated SPECT findings. Nucl Med Commun 2013;34:1107-15.

[18] Cetin M, Kocaman SA, Kiris T, Erdogan T, Canga A, Durakoglugil ME, et al. Absence and resolution of fragmented QRS predict reversible myocardial ischemia with higher probability of ST segment resolution in patients with ST segment elevation myocardial infarction. Korean Circ J 2012;42:674-83. [19] Ozcan S, Cakmak HA, Ikitimur B, Yurtseven E, Stavileci B, Tufekcioglu EY, et al. The prognostic significance of narrow fragmented QRS on admission electrocardiogram in patients hospitalized for decompensated systolic heart failure. Clin Cardiol 2013;36:560-4.

[20] Cheema A, Khalid A, Wimmer A, Bartone C, Chow T, Spertus JA, et al. Fragmented QRS and mortality risk in patients with left ventricular dysfunction. Circ Arrhythm Electrophysiol 2010;3:339-44.

[21] Sha J, Zhang S, Tang M, Chen K, Zhao X, Wang F. Fragmented QRS is associated with all-cause mortality and ventricular arrhythmias in patient with idiopathic dilated cardiomyopathy. Ann Noninvasive Electrocardiol 2011;16:2705 .

[22] John RM, Tedrow UB, Koplan BA, Albert CM, Epstein LM, Sweeney MO, et al. Ventricular arrhythmias and sudden cardiac death. Lancet 2012;380:1520-9.

[23] Belardinelli R, Cianci G, Gigli M, Mazzanti M, Lacalaprice F. Effects of trimetazidine on myocardial perfusion and left ventricular systolic function in type 2 diabetic patients with ischemic cardiomyopathy. J Cardiovasc Pharmacol 2008;51:6115.

[24] Das MK, Suradi H, Maskoun W, Michael MA, Shen C, Peng J, et al. Fragmented wide QRS on a 12-lead ECG: a sign of myocardial scar and poor prognosis. Circ Arrhythm Electrophysiol 2008;1:258-68.

[25] Ozcan F, Turak O, Canpolat U, Avci S, Tok D, Isleyen A, et al. Fragmented QRS predicts the arrhythmic events in patients with heart failure undergoing ICD implantation for primary prophylaxis: more fragments more appropriate ICD shocks. Ann Noninvasive Electrocardiol 2014;19:351-7.

[26] Celikyurt U, Agacdiken A, Sahin T, Al N, Kozdag G, Vural A, et al. Number of leads with fragmented QRS predicts response to cardiac resynchronization therapy. Clin Cardiol 2013;36:369.

[27] Torigoe K, Tamura A, Kawano Y, Shinozaki K, Kotoku M, Kadota J. The number of leads with fragmented QRS is independently associated with cardiac death or hospitalization for heart failure in patients with prior myocardial infarction. $J$ Cardiol 2012;59:36-41. 